\title{
The mode of inheritance of blood pressure levels
}

\author{
R. RONA AND S. A. A. BERESFORD
}

From the Department of Community Medicine, St. Thomas's Hospital Medical School, London

SUMmARY In a sample of 465 families living in a suburb of north-west London the systolic and diastolic blood pressure consistently showed a greater correlation for sib/sib $(0.16$ to 0.28$)$ than for parent/offspring $(0.07$ to $0 \cdot 18)$; this pattern is consistent with the hypothesis of a dominance component in the inheritance of blood pressure assuming there is no difference in the interaction between environment and genes in people of different ages. This assumption was examined by studying the sib/sib correlation according to the age gap between sibs; for diastolic blood pressure this remained almost the same but for systolic blood pressure the correlation tended to diminish as the age gap increased. A dominance component in the inheritance of blood pressure levels could explain the sort of results we have found in this study. However, we cannot ignore the fact that similar results could be obtained if the contribution of the environment within the same generation of relatives differs from that of the environment shared by all relatives.

Few would argue with the statement that genes play an important role in the determination of blood pressure, but what Hamilton et al ${ }^{1}$ stated 20 years ago still applies: "when we come to consider the mode of inheritance, or the extent to which inheritance is a factor in the determination of essential hypertension, we find ourselves on highly uncertain ground'. There have been certain advances in the field in that monogenic inheritance has been largely discounted and a polygenic mode of inheritance together with some environmental participation has become a more attractive theory. ${ }^{2-3}$

Genetic aggregation of blood pressure levels has been found in twin studies and studies of first degree relatives $^{3-9}$ and positive familial association may exist as soon as a month after birth. ${ }^{10}$ It appears that blood pressure clustering in families extends to spouses, which suggests that environmental influences common to family members are partly responsible for familial aggregation of blood pressure levels. Attempts to quantify this in cross-sectional studies have produced contradictory results ${ }^{611-13}$ and in one longitudinal study ${ }^{14}$ no increase of blood pressure clustering was found for increasing length of marriage which might have been expected under the shared environment hypothesis. There has been little or no indication of a significant correlation between parents and adopted children until recently. ${ }^{1516}$ Reanalysis of previously published material, however, has strongly suggested that, after all, adoptees' blood pressure is positively correlated with that of other family members of the same generation, such as another adopted child or a natural sibling. ${ }^{1718}$
No good further evidence on the role of genes in blood pressure has been published in this decade except perhaps by Cavalli-Sforza and Bodmer, ${ }^{19}$ who suggested a genetic model in which dominance plays a fundamental role. They used the regression coefficients found by Miall and Oldham in theip South Wales study. ${ }^{3}$

In this paper we assess the genetic contribution to the determination of blood pressure in a recent set of data and examine whether this contribution varies according to the extent of environmental influence as measured by the differences in age between siblings.

\section{Methods}

A random sample, stratified by family size and social class, was taken from a list of families resident in six electoral wards of Harrow, a suburb of north-west London. The list, compiled for use in a previous study, ${ }^{20}$ was restricted to families with a child born between 1 July 1963 and 30 June 1965 . Of the 568 families sampled, 38 had moved from Harrow and could not be contacted and 29 refused to take part in any more surveys. Five hundred and one families were visited, of whom 465 had at least two children, but in some families information was missing for one or other of the members of interest: mother, father, index child, and eldest sibling in 1970-71. Blood pressure readings were taken twice, on the left arm of the seated subject, using a random-zero sphygmomanometer ${ }^{21}$ to reduce observer bias. For some of the children smaller cuffs were used and adjustments made to measurements obtained. ${ }^{22}$ This is explained in an earlier paper. ${ }^{5}$ 
The mean values of systolic and diastolic (fifth phase) blood pressure and of weight were obtained for each five-year age group for each sex. A standard deviation score (SDS) was then calculated for each mother, father, index child, and eldest sibling by subtracting the mean value of the appropriate age/sex group from the observed measurement, and then dividing by the standard deviation for that age/sex group. Because of the rapid increase in the weight of children with age, especially at puberty, SDS (weight) was recalculated for the index children and siblings under 15 . For each five-year age and sex group a simple linear regression was performed of weight on age. A predicted weight could then be calculated for each child given his exact age, and SDS was calculated thus:

$$
\operatorname{SDS}(\text { weight })=\frac{\text { Observed weight }- \text { predicted weight }}{\begin{array}{l}
\text { Residual mean square for five-year } \\
\text { age and sex group }
\end{array}}
$$

The distributions of SDS (systolic blood pressure) for parents and children and of SDS (diastolic blood pressure) for parents and of SDS(weight) for parents and children were non-Gaussian and very skewed. For the measurements in these groups the SDS were recalculated using the logarithm of the measurement. The resulting SDS distributions were approximately Gaussian. Accordingly, in all cases apart from children's diastolic blood pressure, the SDS were calculated using the log transformation.

Correlation coefficients were calculated to describe the relationship between the scores of first degree relatives. In order to test the relative sizes of the sib/sib correlation and the mother/child or father/child correlation, multiple regressions were performed of index child score on sib score and mother or father score. The partial regression coefficients so obtained were compared using Student's $t$ test (see, for example, Snedecor and Cochrane, ${ }^{23}$ p 391).

The correlation coefficients of raw blood pressure levels of parents/offspring and sib/sib were checked to compare whether the scores used to obtain an approximately Gaussian distribution changed the original relationships between relatives. In practice the changes were very small for all the pairs of relatives.

The variance of the scores was subdivided according to Cavalli-Sforza's and Bodmer's version of a model suggested by Fisher ${ }^{24}$ (see Appendix). The three components were: additive variance as the proportion of the variance due to the difference between the homozygotes summed over all genes; dominance variance as the genetic component due to the fact that a phenotype is nearer to one of the homozygotes; and environmental variance as the remaining part. The correlation coefficients of the SDS of parents and children contain only additive variance, while those of the SDS of index child and eldest sibling contain both additive and dominance variance. As the difference between the sib/sib correlation and the parent/child correlation increases, so the estimate of dominance variance increases. This method of estimating the three components of variance assumes that the environment plays a similar role between sibs as between parents and offspring.

A second set of estimations were calculated adjusting for deviations from random mating, that is, for assortative marriage between parents according to Cavalli-Sforza's and Bodmer's version. This adjustment corrects the additive variance, but assumes that assortative marriage does not alter dominance. In the results, we allow for assortative marriage by presenting the components of variance, taking into account the non-zero blood pressure correlation between parents.

\section{Results}

Table 1 shows SDS correlations for systolic and diastolic blood pressure and weight of mother/child, father/child, sib/sib, mother/father and midparent/child pairs. The correlations of weight and systolic and diastolic blood pressure tend to be larger between sibs than between a parent and his child. The spouse correlations of weight scores were approximately half that of first degree relatives, and the correlations of blood pressure scores tended to be about half those of weight. The sib/sib correlation was significantly greater than the mother/offspring correlation for systolic blood pressure and weight $(P<0.001$ respectively) and the sib/sib correlation was significantly greater than the father/offspring correlation for diastolic blood pressure $(\mathrm{P}<0 \cdot 01)$. The other comparisons between sib/sib and parent/offspring correlations showed similar trends but were non-significant $(0 \cdot 1<\mathrm{P}<0 \cdot 2)$.

The correlation between midparents and index child, which under an additive genetic model is expected to be larger than the sib/sib correlation, proved to be smaller both for systolic and diastolic

Table 1 Pearson correlations in first degree relatives and spouses for blood pressure and weight

\begin{tabular}{lllllll}
\hline Relationship & \multicolumn{2}{c}{ Systolic } & \multicolumn{2}{c}{ Diastolic } & \multicolumn{2}{l}{ Weight } \\
& No. & $r$ & No. & $r$ & No. & $r$ \\
\hline Mother/index child & 450 & 0.149 & 445 & 0.178 & 457 & 0.266 \\
Father/index child & 434 & 0.184 & 419 & 0.068 & 430 & 0.342 \\
Midparent/index child 422 & 0.224 & 416 & 0.160 & 429 & 0.404 \\
Index child/eldest sib & 419 & 0.281 & 408 & 0.232 & 433 & 0.407 \\
Mother/father & 431 & 0.085 & 429 & 0.075 & 432 & 0.147 \\
\hline
\end{tabular}


blood pressure and of the same magnitude for weight. The linear regression coefficients of index child on midparents were almost double the regression coefficients of midparents on index child for both blood pressure and weight, as expected (Table 2). The regression coefficients of weight were substantially greater than those of blood pressure. All the linear regression coefficients were highly significant (at least $P<0 \cdot 01$ ). A second coefficient to test the existence of any curvature of the regression of index child on midparents and of midparents on index child failed to modify significantly the values given by the linear regression coefficients. This was true for all three variables.

Table 2 Linear regression coefficients for child on midparent and midparent on child scores

\begin{tabular}{lllllll}
\hline Relationship & \multicolumn{2}{l}{ Systolic } & \multicolumn{2}{l}{ Diastolic } & \multicolumn{2}{l}{ Weight } \\
& No. & $b$ & No. $b$ & No. $b$ & \\
\hline $\begin{array}{l}\text { Index child on } \\
\text { midparent }\end{array}$ & 422 & 0.303 & 416 & 0.223 & 429 & 0.551 \\
$\begin{array}{c}\text { Midparent on index } \\
\text { child }\end{array}$ & 422 & 0.166 & 416 & 0.115 & 429 & 0.297 \\
\hline
\end{tabular}

The length of marriage of a spouse pair was estimated by adding one year to the age of the eldest child (where both children were fully related to both parents). Table 3 shows that there was no evidence of an increase in spouse correlation with length of marriage. This small association of blood pressure scores may well be a result of assortative marriage. The fact that it is smaller than any of the associations commonly reported for anthropometric measurements ${ }^{25}$ indicates that the blood pressure concordance may be mediated by body build and other related variables.

The stronger association in blood pressure scores between sibs compared with that between parents and offspring could be due to environmental factors acting early in life. The design of this study was not appropriate for a detailed examination of this problem. However, we are able to go some way towards testing this hypothesis by comparing the

Table 3 Pearson correlation of spouse age-adjusted scores

\begin{tabular}{llll}
\hline & \multicolumn{3}{l}{ Length of marriage } \\
\cline { 2 - 4 } & $5-9$ years & $10-19$ years & 20 years and over \\
\hline Systolic BP & 0.081 & 0.081 & $-0 \cdot 180$ \\
Diastolic BP & $(177)$ & $(216)$ & $(31)$ \\
Weight & $0 \cdot 141$ & $0 \cdot 018$ & $0 \cdot 157$ \\
& $(176)$ & $(215)$ & $(31)$ \\
& $0 \cdot 133$ & $0 \cdot 140$ & 0.232 \\
& $(177)$ & $(216)$ & $(31)$ \\
\hline
\end{tabular}

* Age of eldest child + one year.

Numbers of pairs in brackets. correlation of sib/sib scores according to the age gap between the siblings. Our contention is that as the age gap between index child and eldest sib increases, so the years of shared environment in very early life decrease. Table 4 shows the correlations between siblings' blood pressure and weight scores according to the age difference. The correlation for systolic blood pressure slightly decreases as the age gap between sibs increases, whereas the correlation for diastolic blood pressure tends to be almost the same for an age gap of less than 7.5 years and then falls sharply. The correlation of weight tended to remain the same for the first four age gap groups.

Table 4 Pearson correlation coefficient of sib-sib age/sex adjusted score

\begin{tabular}{llllll}
\hline & \multicolumn{6}{c}{ Age gap between index child and eldest sibling } \\
\cline { 2 - 6 } & 0 to & 2.5 to & 5 to & 7.5 to & 10 years and over \\
\hline Systolic BP & 0.352 & 0.280 & 0.180 & 0.243 & 0.222 \\
& $(129)$ & $(141)$ & $(66)$ & $(43)$ & $(44)$ \\
Diastolic BP & 0.244 & 0.264 & 0.329 & 0.088 & -0.009 \\
Weight & $(125)$ & $(136)$ & $(64)$ & $(43)$ & $(44)$ \\
& 0.479 & 0.391 & 0.422 & 0.453 & 0.267 \\
& $(132)$ & $(144)$ & $(73)$ & $(44)$ & $(44)$ \\
\hline
\end{tabular}

Numbers of pairs in brackets.

The partition of variance according to the formulae used by Cavalli-Sforza and Bodmer is shown in Table 5. The major variance component for systolic bloo pressure is the dominance variance, the secondo largest component is the additive variance, and the? smallest proportion is the environmental variance. The pattern is slightly different for diastolic blood pressure and depends on the sex of the parent, but all three components of variance are involved. The environmental variance for weight was almost non-existent.

\section{Discussion}

Our results suggest that sib/sib correlation is consistently higher than parent/offspring correlation

Table 5 Partition of the total variance for blood pressure and weight under assortative marriage

\begin{tabular}{lccc}
\hline Variance component & Systolic & Diastolic & Weight \\
\hline (a) Mother is the parent included for the calculations & \\
& & & \\
Additive & $27.5 \%$ & $33 \cdot 1 \%$ & $46.4 \%$ \\
Dominance & $56.2 \%$ & $24.9 \%$ & $53 \cdot 6 \%$ \\
Environmental & $16.4 \%$ & $42 \cdot 0 \%$ & $0 \cdot 0 \%$ \\
& & \\
(b) Father is the parent included for the calculations & \\
Additive & $33.9 \%$ & $12 \cdot 7 \%$ & $59 \cdot 6 \%$ \\
Dominance & $42.6 \%$ & $67 \cdot 3 \%$ & $33 \cdot 1 \%$ \\
Environmental & $23.5 \%$ & $20 \cdot 1 \%$ & $7.3 \%$ \\
\hline
\end{tabular}


regardless of parent's sex for either systolic or diastolic blood pressure. Several authors have already reported this difference favouring higher correlation for sib/sib. ${ }^{92}$ However, other studies, in particular the Framingham study and the Detroit project studies of blood pressure, have obtained results that do not correspond with our findings, because either the correlation was stronger in the set parent/offspring 4 or the genetic contribution was found to be negligible. ${ }^{27}$ In our analysis we have corrected to a large extent the skewed distribution of blood pressure by the log transformation and therefore it is unlikely that the unequal correlations are artefacts.

The regression coefficients of index child on midparental blood pressure were found to be between 0.22 (diastolic) and 0.30 (systolic) and of midparent on index child $0 \cdot 12$ (diastolic) and $0 \cdot 17$ (systolic). These values are far from the values of 1.0 and 0.5 respectively that are expected under the hypothesis of simple polygenic heredity of blood pressure levels in man.

The partition of variance has been done assuming assortative marriage because most other research has shown a small spouse concordance which is not clearly influenced by length of marriage. Our sample is consistent with this general trend, with the exception perhaps of systolic blood pressure between first and second decades of marriage, where an increase of association was found using a non-parametric method. ${ }^{12}$ Correcting for assortative marriage slightly diminished the additive variance.

The assumption that there is no interaction between genes and environment when using Fisher's partition of variance is difficult to accept. This would mean that the influence of environment among generations is not different from its influence within generations in relatives who are likely to have the same proportion of genes in common. Such interaction would be difficult to estimate. In the Framingham study, Feinleib (see Sackett ${ }^{11}$ ) showed that with a longitudinal design the correlation of adult sibs living apart does not decrease in time whatever the sex of the pair; this indicates that, for adult populations, environment plays little part in changing previous patterns of blood pressure. The question is, to what extent does environment play a role early in life?

The differences between blood pressure correlations for sib/sib and for parent/offspring are unlikely to be explained by the hypothesis that genes come into action at different stages of development, since similar differences between correlations (as estimated by regression coefficients) were obtained in the study by Miall and Oldham in the two adult generations. ${ }^{28}$
A genuine dominance effect has practical as well as theoretical implications; its existence would imply that a small number of genes are responsible in the determination of blood pressure. ${ }^{19}$ Genetic polymorphism at a few loci could be responsible for a large proportion of the variance in blood pressure. From a practical point of view Hunt ${ }^{29}$ suggested that dominance decreases the precision by which offsprings' measurements of, say, blood pressure, can be predicted from the midparent value. The precision will depend on the frequency of the genes that exhibit a dominance effect in the population under surveillance.

There are shortcomings in the dominance hypothesis. Firstly, in two recent papers Annest et $a l^{17} 18$ showed a distinct contribution of environment within the same generation in contrast to the effects of environment shared by all members of the household. Thus parent/adoptee correlations were smaller than the adoptee/adoptee or natural child/adoptee correlations in adoptive families. These results would challenge an assumption that the environment plays a similar role between sibs as between parents and offspring, on which the set of formulae by Cavalli-Sforza and Bodmer are based. Secondly, it is difficult to accept that blood pressure, which is controlled by many hormones and tissue structures, can be dependent on a few genes.

The partition of variance for weight has been presented as an example of a variable which has a similarly skewed distribution to blood pressure and is also known to be associated with it. ${ }^{6}$ The sib/sib and parent/offspring correlations of weight show the same dominance effect as first degree relative correlations for blood pressure. The hypothesis that common genes have a similar mechanism in the determination of each trait is attractive but speculative at this stage. In the exploratory stages of the analysis we did not allow for the rapid increase of weight in childhood and adolescence; the partition of variance showed no dominance component while the environmental component represented almost 50\% of the variation. A slight correction to the correlations of each pair of first degree relatives completely changes the distribution of the genetic and environmental components of the variance. We think that the procedure does not provide a robust estimate of each component of variance. However, our results present strong evidence that the sib/sib correlations tend to be greater than the parent/offspring correlation and this tendency does not represent random fluctuation.

With the many weaknesses that the hypothesis of dominance may still have, it seems attractive to explore the tendency of sib/sib association to be greater than parent/offspring both in exclusively 
adult populations and in adult/child populations. The historical controversy between Pickering ${ }^{2}$ and Platt ${ }^{30}$ has not exhausted the alternative mechanisms in blood pressure level determination. Although Platt's contention is not sustained by most recent research there are other hypotheses that should be explored, ranging from those holding the eclectic view that blood pressure level has different determinants in different populations, ${ }^{27}$ through those advocating genetic determination by additive genes, to those presenting evidence for a dominance component in the variability of blood pressure. The above mentioned mechanisms do not exclude an environmental effect.

Our results have important practical implications: the likelihood of finding new cases of high blood pressure in the family of a known hypertensive would probably be greater among his sibs than among parents and offspring.

We thank Professor W. W. Holland, Miss A. Edwards, and Mrs. A. Childs. This work was supported in part by a grant from the Department of Health and Social Security.

Reprints from Dr. R. Rona, Department of Community Medicine, St. Thomas's Hospital Medical School, London SE1 7EH.

\section{Appendix}

The partition of variance for first degree relatives into three components, additive, dominance, and environmental variance, as given by Cavalli-Sforza and Bodmer, ${ }^{19}$ is based on the fact that parents/offspring correlation $\left(r_{P / O}\right)$ contributes only to the additive variance:

$$
r_{P / O}=\frac{1 / 2 V_{A}}{V_{A}+V_{D}+V_{E}}=\frac{1 / 2 V_{A}}{V_{P}}
$$

while the sib/sib correlation ( $\left.\mathrm{r}_{\mathrm{S} / \mathrm{S}}\right)$ contains both additive and dominance components

$$
r_{S / S}=\frac{1 / 2 V_{A}+1 / 4 V_{D}}{V_{P}}
$$

The total phenotypic variance $\left(V_{P}\right)$ is equal to the sum of additive variance $\left(V_{A}\right)$, dominance variance $\left(V_{D}\right)$, and environmental variance $\left(V_{E}\right)$. Solving (1) and (2) for $V_{A}$ and $V_{D}$ in units of $V_{P}$ gives

$$
\begin{aligned}
& \frac{V_{A}}{V_{P}}=2 r_{P / O} \\
& \frac{V_{D}}{V_{P}}=4\left(r_{S / S}-r_{P / O}\right) \\
& \frac{V_{E}}{V_{P}}=1-\left(V_{A}+V_{D}\right)
\end{aligned}
$$

Assortative mating will modify the components of variance in such a way that under a large number of genes

$$
\hat{\mathbf{V}}_{\mathbf{A}} \sim \frac{\mathbf{V}_{\mathbf{A}}}{1-\hat{\mathbf{A}}}
$$

where

$$
\hat{A}=\frac{\hat{V}_{A}}{\hat{V}_{P}} \times r_{F / M}
$$

Thus $\hat{A}$ represents $V_{A}$ modified by the correlation between father and mother $\left(r_{F / M}\right)$. Under the assumption that $V_{D}$ is not affected by assortative mating, the parent/offspring correlation is

$$
r_{P / O}=\frac{\left(1+r_{F / M}\right) \hat{V}_{A}}{2 \hat{V}_{P}}
$$

The correlation sib/sib is

$$
r_{S / S}=1 / 4 \frac{\hat{V}_{A}+V_{D}}{\hat{V}_{P}}\left[1+\frac{\hat{V}_{A}}{\hat{V}_{A}+V_{D}}(1+2 \hat{A})\right]
$$

and using (8) and (9), the components of variance are

$$
\begin{aligned}
& \frac{\hat{V}_{A}}{\hat{V}_{P}}=\frac{2 r_{P / O}}{1+r_{F / M}} \\
& \frac{V_{D}}{\hat{V}_{P}}=4 r_{S / S}-\frac{4 r_{P / O}}{\left(1+r_{F / M}\right)}\left[1+\frac{r_{F / M} r_{P / O}}{\left(1+r_{F / M}\right)}\right]
\end{aligned}
$$

and

$$
\frac{\hat{V}_{E}}{\hat{V}_{P}}=1-\frac{\hat{V}_{A}}{\hat{V}_{P}}-\frac{V_{D}}{\hat{V}_{P}}
$$

can be calculated using (10) and (11).

\section{References}

${ }^{1}$ Hamilton M, Pickering GW, Fraser Roberts JA, Sowry GSC. The etiology of essential hypertension. 4-The role of inheritance. Clin Sci 1954; 13: 273-304.

${ }^{2}$ Pickering GW. The inheritance of arterial pressure. In: High Blood Pressure. 2nd edn. London: Churchill, 1968.

${ }^{3}$ Miall WE, Oldham PD. The heredity factor in arterial blood pressure. $\mathrm{Br}$ Med J 1963; i: 75-80.

${ }^{4}$ Feinleib M, Garrison R, Borham N, Rosenman R, Christian J. Studies of hypertension in twins. In: Paul O, ed. Epidemiology and control of hypertension. Second International Symposium on the Epidemiology of Hypertension. New York, London: Symposia Specialist, 1974.

${ }^{5}$ Holland WW, Beresford SAA. Factors influencing blood pressure in children. In: Paul O, ed. Epidemiology and control of hypertension. Second International Symposium on the Epidemiology of Hypertension. New York, London: Symposia Specialist, 1974.

${ }^{6}$ Hayes CG, Tyroler HA, Cassel JC. Family aggregation of blood pressure in Evans Country, Georgia. Arch Intern Med 1971; 128: 965-75.

${ }^{7}$ Johnson BC, Epstein FH, Kjelsberg MO. Distributions and familial studies of blood pressure and serum cholesterol levels in a total community-Tecumseh, Michigan. J Chron Dis 1965; 18: 147-59.

${ }^{8}$ Winkelstein W, Kantor S, Ibrahim M, Sackett DL. Familial aggregation of blood pressure. JAMA 1966; 195: $160-2$. 
${ }^{9}$ Zinner SH, Levy PS, Kass EH. Familial aggregation of blood pressure in childhood. N Engl J Med 1971; 284: 401-4.

${ }^{10}$ Hennekens $\mathrm{ChH}$, Jesse MJ, Klein BE, Gourley JE, Blumenthal S. Aggregation of blood pressure in infants and their siblings. Am J Epidemiol 1976; 103: 457-63.

${ }^{11}$ Sackett D. Studies of blood pressure in spouses. In: Paul $\mathrm{O}$, ed. Epidemiology and control of hypertension. Second International Symposium on the Epidemiology of Hypertension. New York, London: Symposia Specialist, 1974.

${ }^{12}$ Beresford SAA. Concordance of blood pressure in spouses. Rev Epidemiol Sante Publique 1976; 24: 395-403.

${ }^{13}$ Winkelstein W, Kantor S, Ibrahim MA, Sackett DL. Remarks on the analysis of familial aggregation in the Alameda County Blood Pressure Study. Am J Epidemiol 1969; 89: 615-8.

${ }^{14}$ Sackett DL, Anderson GD, Milner R, Feinleib M, Kannel WB. Concordance for coronary risk factors among spouses. Circulation 1975; 52: 589-95.

${ }^{15}$ Biron P, Mongeau JB, Bertrand D. Familial aggregation of blood pressure in adopted and natural children. In: Paul O, ed. Epidemiology and control of hypertension. Second International Symposium on the Epidemiology of Hypertension. New York, London: Symposia Specialist, 1974.

${ }^{16}$ Higgins M, Cole P, Garn S, Keller J. Familial resemblance in blood pressure. Cardiovascular Disease Epidemiology 1977; (Newsletter) 22: 24.

${ }^{17}$ Annest JL, Sing ChF, Biron P, Mongeau J. Familial aggregation of blood pressure and weight in adoptive families. I-Comparisons of blood pressure and weight statistics among families with adopted, natural or both natural and adopted children. Am J Epidemiol 1979; 110: 479-91.
${ }^{18}$ Annest JC, Sing ChF, Biron P, Mongeau J. Familial aggregation of blood pressure and weight in adoptive families. II-Estimation of the relative contributions of genetic and common environmental factors to blood pressure correlations between family members. Am J Epidemiol 1979; 110: 492-503.

${ }^{19}$ Cavalli-Sforza LL, Bodmer WF. The Genetics of Human Populations. San Francisco: W. H. Freeman, 1971.

${ }^{20}$ Holland WW, Kasap HS, Colley JRT, Cormack W. Respiratory symptoms and ventilatory function: A family study. Br J Prev Soc Med 1969; 23: 77-84.

${ }^{21}$ Wright BM, Dore CF. A random-zero sphygmomanometer. Lancet 1970; i: 337-8.

${ }^{22}$ Long M, Dunlop JR, Holland WW. Blood pressure recording in children. Arch Dis Child 1971;46: 636-40.

${ }^{23}$ Snedecor GW, Cochran G. Statistical Methods. 6th edn. Iowa: Iowa State University Press, 1967.

${ }^{24}$ Fisher RA. Correlation between relatives in supposition of mendelian inheritance. Transactions of the Royal Society of Edinburgh 1919; 52: 399-433.

${ }^{25}$ Spuhler JN. Assortative mating with respect to physical characteristics. Eugenics Quarterly 1968; 15: 128-40.

${ }^{26}$ Cruz-Coke R, Donoso H, Barrera R. Genetic ecology of hypertension. Clin Sci Mol Med 1973; 45: 55-65.

${ }^{27}$ Chakraborty R, Schull WJ, Harburg E, Shork MA, Roeper P. Heredity, stress and blood pressure, a family set method. V-Heritability estimates. J Chron Dis 1977; 30: 683-99.

${ }^{28}$ Miall WE, Oldham PD. Factors influencing arterial blood pressure in the general population. Clin Sci 1958; 17: $409-44$.

${ }^{29}$ Hunt EE. The developmental genetics of man. In: Falkner F, ed. Human Development. Philadelphia, London: WB Saunders, 1966.

${ }^{30}$ Platt R. Essential hypertension: Incidence, course and heredity. Ann Intern Med 1961; 55: 1-11. 